\begin{tabular}{ll}
789 \\
JOB OURNAL OF \\
\hline
\end{tabular}

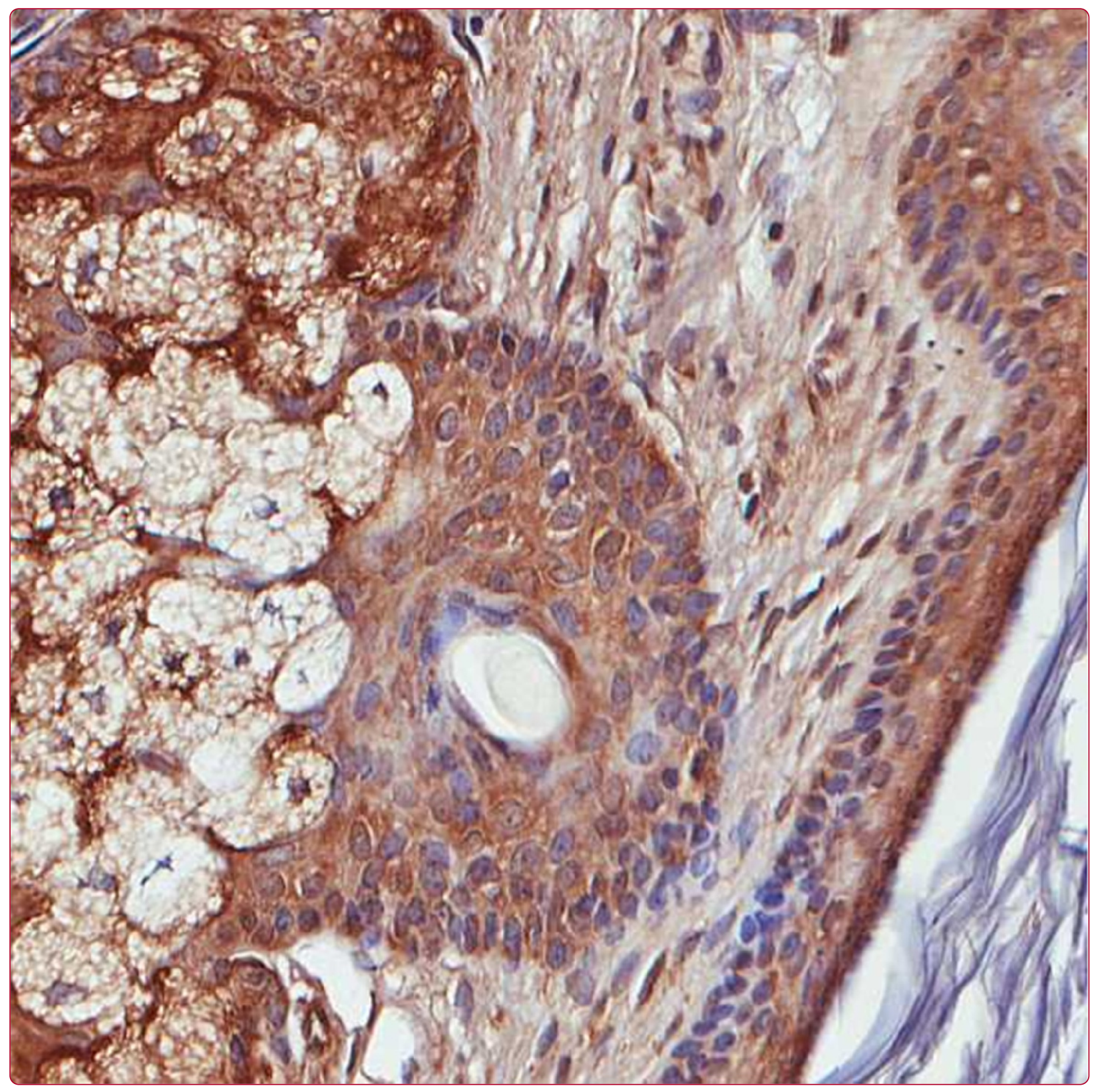

Fractalkine receptor is expressed in mature ovarian teratomas and required for epidermal lineage differentiation

Rooper et al. 


\title{
Fractalkine receptor is expressed in mature ovarian teratomas and required for epidermal lineage differentiation
}

\author{
Lisa Rooper ${ }^{1}$, Hilal Gurler², Andre A Kajdacsy-Balla ${ }^{1}$ and Maria V Barbolina ${ }^{2 *}$
}

\begin{abstract}
Background: The goal of this study was to determine a predominant cell type expressing fractalkine receptor $\left(C X_{3} C R 1\right)$ in mature ovarian teratomas and to establish functional significance of its expression in cell differentiation.

Methods: Specimens of ovarian teratoma and human fetal tissues were analyzed by immunohistochemistry for $\mathrm{CX}_{3}$ CR1 expression. Ovarian teratocarcinoma cell line PA-1 was used as a model for cell differentiation.

Results: We found that the majority of the specimens contained $C X_{3} C R 1$-positive cells of epidermal lineage. Skin keratinocytes in fetal tissues were also $C X_{3} C R 1$ - positive. PA-1 cells with downregulated $C X_{3} C R 1$ failed to express a skin keratinocyte marker cytokeratin 14 when cultured on Matrigel in the presence of a morphogen, bone morphogenic protein 4 (BMP-4), as compared to those expressing scrambled shRNA.

Conclusions: Here we demonstrate that $\mathrm{CX}_{3} \mathrm{CR} 1$ is expressed in both normally (fetal skin) and abnormally (ovarian teratoma) differentiated keratinocytes and is required for cell differentiation into epidermal lineage.
\end{abstract}

Keywords: Mature ovarian teratoma, Fractalkine receptor, Cell differentiation

\section{Background}

Ovarian teratoma (dermoid cyst) is a benign tumor originating in germ cells. This tumor is one of the most common germ cell tumors and accounts for up to $20 \%$ of all ovarian cysts [1]. Ovarian teratoma is the most common tumor associated with pregnancy [2]. Generally, ovarian teratomas are cured by a complete surgical resection; however, some cases could give rise to a malignant disease [3,4]. Ovarian teratomas could be of both homozygous and heterozygous origin, as well as the mixture of the two, and it has been previously suggested that several mechanisms could lead to their development, including failure of meiosis I, failure of meiosis II, or duplication of a mature ovum [5]. Mature ovarian teratomas often contain a mixture of fully differentiated tissues from all three cell layers, ectodermal, endodermal, and mesodermal, resulting in formation of skin,

\footnotetext{
*Correspondence: mvb@uic.edu

${ }^{2}$ Biopharmaceutical Sciences, University of Illinois at Chicago, 833 S Wood Str, Chicago, IL 60612, USA

Full list of author information is available at the end of the article
}

hair follicle, sebaceous gland, bone, teeth, thyroid, and other cell types [6,7].

Our previous data obtained using a limited number of subjects suggested that a chemokine receptor fractalkine $\left(\mathrm{CX}_{3} \mathrm{CR} 1\right)$ is expressed in ovarian teratomas [8]. $\mathrm{CX}_{3} \mathrm{CR} 1$ is a $\mathrm{G}$ protein-coupled receptor with multiple functions in both the normal state and disease $[9,10]$. $\mathrm{CX}_{3} \mathrm{CR} 1$ is activated by its highly specific ligand fractalkine $\left(\mathrm{CX}_{3} \mathrm{CL} 1\right)$ [11]. It has been shown that $\mathrm{CX}_{3} \mathrm{CL} 1 / \mathrm{CX}_{3} \mathrm{CR} 1$ axis plays a role in differentiation of osteoclasts [12] and development of dendritic cells [13], but its role in skin differentiation has not been described.

The goal of this study was to determine the predominant type of $\mathrm{CX}_{3} \mathrm{CR} 1$-positive cells in ovarian teratomas. We also aimed to determine whether the same cell type (s) are $\mathrm{CX}_{3} \mathrm{CR} 1$-positive during the normal development. Lastly, using a cell culture model, we intended to establish whether $\mathrm{CX}_{3} \mathrm{CR} 1$ expression is required for cell differentiation. 
Table 1 Expression of CX3CR1 in human specimens of ovarian teratoma

\begin{tabular}{|c|c|c|c|c|c|c|c|c|c|c|c|}
\hline Position & Number & Sex & Age & Organ & Pathology & Grade & Type & Tissue type & Intensity* & Location** & Percentage $^{* * *}$ \\
\hline $\mathrm{A} 1$ & 1 & $\mathrm{~F}$ & 28 & Ovary & Mature teratoma & - & Benign & Sebaceous & 2.5 & $C$ & 100 \\
\hline $\mathrm{A} 1$ & 1 & $\mathrm{~F}$ & 28 & Ovary & Mature teratoma & - & Benign & Apocrine & 1 & C & 10 \\
\hline$A 2$ & 2 & $\mathrm{~F}$ & 24 & Ovary & Mature teratoma & - & Benign & Skin & 2 & C & 20 \\
\hline A2 & 2 & $\mathrm{~F}$ & 24 & Ovary & Mature teratoma & - & Benign & Lymphocytes & 3 & N & 90 \\
\hline A3 & 3 & $\mathrm{~F}$ & 26 & Ovary & Mature teratoma & - & Benign & NR & & & \\
\hline A4 & 4 & $\mathrm{~F}$ & 33 & Ovary & Mature teratoma & - & Benign & Skin & 3 & C & 100 \\
\hline A4 & 4 & $\mathrm{~F}$ & 33 & Ovary & Mature teratoma & - & Benign & Sebaceous & 3 & C & 100 \\
\hline A5 & 5 & $\mathrm{~F}$ & 48 & Ovary & Mature teratoma & - & Benign & Follicle & 3 & C & 100 \\
\hline A6 & 6 & $\mathrm{~F}$ & 19 & Ovary & Mature teratoma & - & Benign & Skin & 3 & C & 100 \\
\hline A6 & 6 & $\mathrm{~F}$ & 19 & Ovary & Mature teratoma & - & Benign & Endothelium & 1.5 & C & 90 \\
\hline A7 & 7 & $\mathrm{~F}$ & 52 & Ovary & Mature teratoma & - & Benign & Skin & 2.5 & C & 100 \\
\hline A7 & 7 & $\mathrm{~F}$ & 52 & Ovary & Mature teratoma & - & Benign & Sebaceous & 2.5 & C & 100 \\
\hline A8 & 8 & $\mathrm{~F}$ & 27 & Ovary & Mature teratoma & - & Benign & Skin & 2 & C & 100 \\
\hline A8 & 8 & $\mathrm{~F}$ & 27 & Ovary & Mature teratoma & - & Benign & Apocrine & 3 & C & 100 \\
\hline A8 & 8 & $\mathrm{~F}$ & 27 & Ovary & Mature teratoma & - & Benign & Lymph & 3 & NM & 50 \\
\hline A9 & 9 & $\mathrm{~F}$ & 22 & Ovary & Mature teratoma & - & Benign & Sebaceous & 3 & C & 100 \\
\hline A10 & 10 & $\mathrm{~F}$ & 54 & Ovary & Mature teratoma & - & Benign & Skin & 2 & C & 100 \\
\hline $\mathrm{A} 10$ & 10 & $\mathrm{~F}$ & 54 & Ovary & Mature teratoma & - & Benign & Sebaceous & 2.5 & C & 100 \\
\hline B1 & 11 & $\mathrm{~F}$ & 58 & Ovary & Mature teratoma & - & Benign & Adipose & 1 & C & 80 \\
\hline B1 & 11 & $\mathrm{~F}$ & 58 & Ovary & Mature teratoma & - & Benign & Adipose & 3 & M & 40 \\
\hline B2 & 12 & $\mathrm{~F}$ & 32 & Ovary & Mature teratoma & - & Benign & Skin & 2 & C & 100 \\
\hline B2 & 12 & $\mathrm{~F}$ & 32 & Ovary & Mature teratoma & - & Benign & Follicle & 2.5 & C & 100 \\
\hline B2 & 12 & $\mathrm{~F}$ & 32 & Ovary & Mature teratoma & - & Benign & Sebaceous & 3 & C & 100 \\
\hline B3 & 13 & $\mathrm{~F}$ & 28 & Ovary & Mature teratoma & - & Benign & $N R$ & & & \\
\hline B4 & 14 & $\mathrm{~F}$ & 40 & Ovary & Mature teratoma & - & Benign & Adipose & 1 & C & 60 \\
\hline B4 & 14 & $\mathrm{~F}$ & 40 & Ovary & Mature teratoma & - & Benign & Adipose & 3 & M & 40 \\
\hline B5 & 15 & $\mathrm{~F}$ & 26 & Ovary & Mature teratoma & - & Benign & Sebaceous & 3 & C & 70 \\
\hline B5 & 15 & $\mathrm{~F}$ & 26 & Ovary & Mature teratoma & - & Benign & Apocrine & 3 & C & 100 \\
\hline B6 & 16 & $\mathrm{~F}$ & 39 & Ovary & Mature teratoma & - & Benign & Endothelium & 3 & C & 100 \\
\hline B6 & 16 & $\mathrm{~F}$ & 39 & Ovary & Mature teratoma & - & Benign & Lymphocytes & 3 & N & 70 \\
\hline B7 & 17 & $\mathrm{~F}$ & 23 & Ovary & Mature teratoma & - & Benign & Lymphocytes & 3 & $N$ & 40 \\
\hline B7 & 17 & $\mathrm{~F}$ & 23 & Ovary & Mature teratoma & - & Benign & Apocrine & 2.5 & C & 30 \\
\hline B8 & 18 & $\mathrm{~F}$ & 32 & Ovary & Mature teratoma & - & Benign & Sebaceous & 3 & C & 100 \\
\hline B8 & 18 & $\mathrm{~F}$ & 32 & Ovary & Mature teratoma & - & Benign & Skin & 3 & C & 100 \\
\hline B8 & 18 & $\mathrm{~F}$ & 32 & Ovary & Mature teratoma & - & Benign & Thyroid & 3 & C & 80 \\
\hline B9 & 19 & $\mathrm{~F}$ & 29 & Ovary & Mature teratoma & - & Benign & Thyroid & 3 & C & 80 \\
\hline B10 & 20 & $\mathrm{~F}$ & 30 & Ovary & Mature teratoma & - & Benign & Follicle & 2 & C & 100 \\
\hline $\mathrm{C} 1$ & 21 & $\mathrm{~F}$ & 40 & Ovary & Mature teratoma & - & Benign & Sebaceous & 3 & C & 100 \\
\hline $\mathrm{C} 1$ & 21 & $\mathrm{~F}$ & 40 & Ovary & Mature teratoma & - & Benign & Skin & 1.5 & C & 100 \\
\hline $\mathrm{C} 2$ & 22 & $\mathrm{~F}$ & 35 & Ovary & Mature teratoma & - & Benign & Sebaceous & 3 & C & 100 \\
\hline $\mathrm{C} 2$ & 22 & $\mathrm{~F}$ & 35 & Ovary & Mature teratoma & - & Benign & Skin & 3 & C & 100 \\
\hline $\mathrm{C} 3$ & 23 & $\mathrm{~F}$ & 49 & Ovary & Mature teratoma & - & Benign & Lymphocytes & 3 & N & 10 \\
\hline C4 & 24 & $\mathrm{~F}$ & 51 & Ovary & Mature teratoma & - & Benign & Skin & 3 & C & 100 \\
\hline C5 & 25 & $\mathrm{~F}$ & 22 & Ovary & Mature teratoma & - & Benign & Skin & 2 & C & 100 \\
\hline
\end{tabular}


Table 1 Expression of CX3CR1 in human specimens of ovarian teratoma (Continued)

\begin{tabular}{|c|c|c|c|c|c|c|c|c|c|c|c|}
\hline $\mathrm{C5}$ & 25 & $\mathrm{~F}$ & 22 & Ovary & Mature teratoma & - & Benign & Lymphocytes & 2 & $N$ & 10 \\
\hline $\mathrm{C} 6$ & 26 & $\mathrm{~F}$ & 69 & Ovary & Mature teratoma & - & Benign & NR & & & \\
\hline $\mathrm{C7}$ & 27 & $\mathrm{~F}$ & 35 & Ovary & Mature teratoma & - & Benign & Sebaceous & 3 & C & 100 \\
\hline $\mathrm{C7}$ & 27 & $\mathrm{~F}$ & 35 & Ovary & Mature teratoma & - & Benign & Follicle & 2 & C & 100 \\
\hline $\mathrm{C7}$ & 27 & $\mathrm{~F}$ & 35 & Ovary & Mature teratoma & - & Benign & Apocrine & 2.5 & C & 90 \\
\hline $\mathrm{C} 8$ & 28 & $\mathrm{~F}$ & 8 & Ovary & Mature teratoma & - & Benign & Apocrine & 2.5 & M & 100 \\
\hline $\mathrm{C} 8$ & 28 & $\mathrm{~F}$ & 8 & Ovary & Mature teratoma & - & Benign & Apocrine & 1 & C & 50 \\
\hline C9 & 29 & $\mathrm{~F}$ & 23 & Ovary & Mature teratoma & - & Benign & Sebaceous & 3 & C & 100 \\
\hline C9 & 29 & $\mathrm{~F}$ & 23 & Ovary & Mature teratoma & - & Benign & Skin & 3 & C & 90 \\
\hline $\mathrm{C} 10$ & 30 & $\mathrm{~F}$ & 26 & Ovary & Mature teratoma & - & Benign & NR & & & \\
\hline D1 & 31 & F & 35 & Ovary & Mature teratoma & - & Benign & Sebaceous & 3 & C & 100 \\
\hline D1 & 31 & $\mathrm{~F}$ & 35 & Ovary & Mature teratoma & - & Benign & Apocrine & 3 & C & 60 \\
\hline D2 & 32 & $\mathrm{~F}$ & 47 & Ovary & Mature teratoma & - & Benign & Skin & 2 & C & 100 \\
\hline D2 & 32 & $\mathrm{~F}$ & 47 & Ovary & Mature teratoma & - & Benign & Sebaceous & 3 & C & 100 \\
\hline D3 & 33 & $\mathrm{~F}$ & 56 & Ovary & Mature teratoma & - & Benign & Adipose & 1 & C & 60 \\
\hline D3 & 33 & $\mathrm{~F}$ & 56 & Ovary & Mature teratoma & - & Benign & Adipose & 3 & M & 40 \\
\hline D3 & 33 & $\mathrm{~F}$ & 56 & Ovary & Mature teratoma & - & Benign & Apocrine & 3 & C & 70 \\
\hline D4 & 34 & $\mathrm{~F}$ & 40 & Ovary & Mature teratoma & - & Benign & $N R$ & & & \\
\hline D5 & 35 & $\mathrm{~F}$ & 32 & Ovary & Mature teratoma & - & Benign & Skin & 3 & C & 100 \\
\hline D5 & 35 & $\mathrm{~F}$ & 32 & Ovary & Mature teratoma & - & Benign & Sebaceous & 3 & C & 100 \\
\hline D6 & 36 & $F$ & 12 & Ovary & Mature teratoma & - & Benign & Lymphocyte & 3 & $\mathrm{~N}$ & 40 \\
\hline D6 & 36 & $\mathrm{~F}$ & 12 & Ovary & Mature teratoma & - & Benign & Apocrine & 3 & C & 70 \\
\hline D7 & 37 & $\mathrm{~F}$ & 43 & Ovary & Mature teratoma & - & Benign & $N R$ & & & \\
\hline D8 & 38 & F & 27 & Ovary & Mature teratoma & - & Benign & Skin & 2 & C & 100 \\
\hline D9 & 39 & $\mathrm{~F}$ & 22 & Ovary & Mature teratoma & - & Benign & Sebaceous & 3 & C & 100 \\
\hline D9 & 39 & $\mathrm{~F}$ & 22 & Ovary & Mature teratoma & - & Benign & Eccrine & 1 & C & 30 \\
\hline D10 & 40 & $\mathrm{~F}$ & 40 & Ovary & Mature teratoma & - & Benign & Adipose & 1 & C & 60 \\
\hline D10 & 40 & $\mathrm{~F}$ & 40 & Ovary & Mature teratoma & - & Benign & Adipose & 3 & M & 30 \\
\hline D10 & 40 & $\mathrm{~F}$ & 40 & Ovary & Mature teratoma & - & Benign & Skin & 3 & C & 100 \\
\hline E1 & 41 & $\mathrm{~F}$ & 52 & Ovary & Mature teratoma & - & Benign & $\begin{array}{l}\text { Respiratory } \\
\text { epithelium }\end{array}$ & 2.5 & C & 80 \\
\hline E2 & 42 & $\mathrm{~F}$ & 23 & Ovary & Mature teratoma & - & Benign & Sebaceous & 3 & C & 100 \\
\hline E2 & 42 & $\mathrm{~F}$ & 23 & Ovary & Mature teratoma & - & Benign & Thyroid & 1.5 & C & 70 \\
\hline E2 & 42 & $\mathrm{~F}$ & 23 & Ovary & Mature teratoma & - & Benign & Apocrine & 2 & C & 70 \\
\hline E3 & 43 & $\mathrm{~F}$ & 23 & Ovary & Mature teratoma & - & Benign & $\begin{array}{l}\text { Respiratory } \\
\text { epithelium }\end{array}$ & 2 & C & 50 \\
\hline E3 & 43 & $\mathrm{~F}$ & 23 & Ovary & Mature teratoma & - & Benign & Lymphocytes & 3 & $\mathrm{~N}$ & 20 \\
\hline E4 & 44 & F & 47 & Ovary & Mature teratoma & - & Benign & Skin & 3 & C & 100 \\
\hline E4 & 44 & $\mathrm{~F}$ & 47 & Ovary & Mature teratoma & - & Benign & Nerve & 3 & NM & 80 \\
\hline E4 & 44 & $\mathrm{~F}$ & 47 & Ovary & Mature teratoma & - & Benign & Nerve & 2 & C & 70 \\
\hline E5 & 45 & $\mathrm{~F}$ & 45 & Ovary & Mature teratoma & - & Benign & Skin & 3 & C & 100 \\
\hline E6 & 46 & $\mathrm{~F}$ & 33 & Ovary & Mature teratoma & - & Benign & Sebaceous & 3 & C & 100 \\
\hline E6 & 46 & $\mathrm{~F}$ & 33 & Ovary & Mature teratoma & - & Benign & $\begin{array}{l}\text { Respiratory } \\
\text { epithelium }\end{array}$ & 1.5 & C & 100 \\
\hline E6 & 46 & F & 33 & Ovary & Mature teratoma & - & Benign & Apocrine & 2 & C & 70 \\
\hline E6 & 46 & $\mathrm{~F}$ & 33 & Ovary & Mature teratoma & - & Benign & Lymphocytes & 3 & $\mathrm{~N}$ & 5 \\
\hline
\end{tabular}


Table 1 Expression of CX3CR1 in human specimens of ovarian teratoma (Continued)

\begin{tabular}{|c|c|c|c|c|c|c|c|c|c|c|c|}
\hline E7 & 47 & $\mathrm{~F}$ & 27 & Ovary & Mature teratoma & - & Benign & Sebaceous & 2.5 & $C$ & 100 \\
\hline E7 & 47 & $\mathrm{~F}$ & 27 & Ovary & Mature teratoma & - & Benign & Lymphocytes & 3 & N & 30 \\
\hline E7 & 47 & $\mathrm{~F}$ & 27 & Ovary & Mature teratoma & - & Benign & Skin & 2 & C & 90 \\
\hline E8 & 48 & $F$ & 28 & Ovary & Mature teratoma & - & Benign & Adipose & 1 & C & 100 \\
\hline E8 & 48 & $\mathrm{~F}$ & 28 & Ovary & Mature teratoma & - & Benign & Adipose & 3 & M & 50 \\
\hline E8 & 48 & $\mathrm{~F}$ & 28 & Ovary & Mature teratoma & - & Benign & Follicle & 3 & C & 90 \\
\hline E9 & 49 & $\mathrm{~F}$ & 24 & Ovary & Mature teratoma & - & Benign & NR & & & \\
\hline E10 & 50 & $F$ & 22 & Ovary & Mature teratoma & - & Benign & Skin & 3 & C & 100 \\
\hline $\mathrm{F} 1$ & 51 & $\mathrm{~F}$ & 27 & Ovary & $\begin{array}{l}\text { Mature teratoma } \\
\text { (ovary tissue) }\end{array}$ & - & Benign & NR & & & \\
\hline $\mathrm{F} 2$ & 52 & $F$ & 32 & Ovary & Mature teratoma & - & Benign & Lymphocytes & 3 & N & 100 \\
\hline F3 & 53 & $\mathrm{~F}$ & 42 & Ovary & Mature teratoma & - & Benign & $N R$ & & & \\
\hline $\mathrm{F} 4$ & 54 & $F$ & 30 & Ovary & Mature teratoma & - & Benign & Adipose & 1 & C & 30 \\
\hline $\mathrm{F} 4$ & 54 & $\mathrm{~F}$ & 30 & Ovary & Mature teratoma & - & Benign & Adipose & 3 & M & 15 \\
\hline F5 & 55 & $\mathrm{~F}$ & 38 & Ovary & Mature teratoma & - & Benign & Sebaceous & 3 & C & 100 \\
\hline F5 & 55 & $\mathrm{~F}$ & 38 & Ovary & Mature teratoma & - & Benign & Skin & 2.5 & C & 90 \\
\hline F5 & 55 & $\mathrm{~F}$ & 38 & Ovary & Mature teratoma & - & Benign & Lymphocytes & 3 & N & 10 \\
\hline F6 & 56 & $\mathrm{~F}$ & 36 & Ovary & Mature teratoma & - & Benign & Sebaceous & 3 & C & 100 \\
\hline F6 & 56 & $\mathrm{~F}$ & 36 & Ovary & Mature teratoma & - & Benign & Skin & 3 & C & 100 \\
\hline F7 & 57 & $\mathrm{~F}$ & 25 & Ovary & Mature teratoma & - & Benign & Skin & 3 & C & 100 \\
\hline F7 & 57 & $\mathrm{~F}$ & 25 & Ovary & Mature teratoma & - & Benign & Lymphocytes & 3 & N & 90 \\
\hline F8 & 58 & $\mathrm{~F}$ & 39 & Ovary & Mature teratoma & - & Benign & Skin & 3 & C & 100 \\
\hline F8 & 58 & $\mathrm{~F}$ & 39 & Ovary & Mature teratoma & - & Benign & Sebaceous & 3 & C & 100 \\
\hline F9 & 59 & $F$ & 32 & Ovary & Mature teratoma & - & Benign & Sebaceous & 3 & C & 100 \\
\hline F9 & 59 & $\mathrm{~F}$ & 32 & Ovary & Mature teratoma & - & Benign & Skin & 3 & C & 100 \\
\hline F9 & 59 & $F$ & 32 & Ovary & Mature teratoma & - & Benign & Thyroid & 2.5 & C & 50 \\
\hline F10 & 60 & $\mathrm{~F}$ & 29 & Ovary & Mature teratoma & - & Benign & Nerve & 3 & NM & 100 \\
\hline F10 & 60 & $\mathrm{~F}$ & 29 & Ovary & Mature teratoma & - & Benign & Nerve & 2.5 & C & 100 \\
\hline F10 & 60 & $\mathrm{~F}$ & 29 & Ovary & Mature teratoma & - & Benign & Adipose & 1 & C & 10 \\
\hline F10 & 60 & $\mathrm{~F}$ & 29 & Ovary & Mature teratoma & - & Benign & Adipose & 3 & M & 10 \\
\hline F10 & 60 & $\mathrm{~F}$ & 29 & Ovary & Mature teratoma & - & Benign & Follicle & 2 & C & 100 \\
\hline F10 & 60 & $\mathrm{~F}$ & 29 & Ovary & Mature teratoma & - & Benign & Sebaceous & 3 & C & 100 \\
\hline G1 & 61 & $F$ & 32 & Ovary & Mature teratoma & - & Benign & $N R$ & & & \\
\hline G2 & 62 & $\mathrm{~F}$ & 36 & Ovary & Mature teratoma & - & Benign & Adipose & 1 & C & 20 \\
\hline G2 & 62 & $\mathrm{~F}$ & 36 & Ovary & Mature teratoma & - & Benign & Adipose & 3 & M & 10 \\
\hline G3 & 63 & $\mathrm{~F}$ & 29 & Ovary & Mature teratoma & - & Benign & NR & & & \\
\hline G4 & 64 & $F$ & 39 & Ovary & Mature teratoma & - & Benign & Nerve & 3 & NM & 10 \\
\hline G5 & 65 & $\mathrm{~F}$ & 15 & Ovary & Immature teratoma & $\|$ & Malignant & Lymphocytes & 3 & N & 10 \\
\hline G6 & 66 & $\mathrm{~F}$ & 40 & Ovary & Immature teratoma & - & Malignant & $N R$ & & & \\
\hline G7 & 67 & $\mathrm{~F}$ & 29 & Ovary & Immature teratoma & - & Malignant & Immature & 3 & $\mathrm{C} / \mathrm{N}$ & 90 \\
\hline G8 & 68 & $\mathrm{~F}$ & 37 & Ovary & Immature teratoma & - & Malignant & Immature & 2.5 & C & 100 \\
\hline G9 & 69 & $\mathrm{~F}$ & 39 & Ovary & Mature teratoma & - & Benign & Skin & 3 & C & 100 \\
\hline G10 & 70 & $\mathrm{~F}$ & 40 & Ovary & Mature teratoma & - & Benign & Sebaceous & 3 & C & 100 \\
\hline G10 & 70 & $\mathrm{~F}$ & 40 & Ovary & Mature teratoma & - & Benign & Skin & 3 & C & 100 \\
\hline $\mathrm{H} 1$ & 71 & $\mathrm{~F}$ & 49 & Ovary & Mature teratoma & - & Benign & Adipose & 1 & C & 30 \\
\hline
\end{tabular}


Table 1 Expression of CX3CR1 in human specimens of ovarian teratoma (Continued)

\begin{tabular}{|c|c|c|c|c|c|c|c|c|c|c|c|}
\hline $\mathrm{H} 1$ & 71 & $\mathrm{~F}$ & 49 & Ovary & Mature teratoma & - & Benign & Adipose & 3 & M & 10 \\
\hline $\mathrm{H} 2$ & 72 & $\mathrm{~F}$ & 28 & Ovary & Immature teratoma & $\|$ & Malignant & NR & & & \\
\hline $\mathrm{H} 3$ & 73 & $\mathrm{~F}$ & 37 & Ovary & Immature teratoma & - & Malignant & Immature & 1 & $C$ & 70 \\
\hline $\mathrm{H} 4$ & 74 & $\mathrm{~F}$ & 22 & Ovary & Immature teratoma & $\|$ & Malignant & $\begin{array}{l}\text { Respiratory } \\
\text { epithelium }\end{array}$ & 2 & $C$ & 90 \\
\hline $\mathrm{H} 4$ & 74 & $\mathrm{~F}$ & 22 & Ovary & Immature teratoma & $\|$ & Malignant & Lymphocyte & 3 & $N$ & 30 \\
\hline $\mathrm{H} 5$ & 75 & $\mathrm{~F}$ & 28 & Ovary & Immature teratoma & $\|$ & Malignant & Adipose & 1 & $C$ & 100 \\
\hline $\mathrm{H} 5$ & 75 & $\mathrm{~F}$ & 28 & Ovary & Immature teratoma & $\|$ & Malignant & $\begin{array}{l}\text { Respiratory } \\
\text { epithelium }\end{array}$ & 2.5 & $C$ & 100 \\
\hline $\mathrm{H} 5$ & 75 & $\mathrm{~F}$ & 28 & Ovary & Immature teratoma & $\|$ & Malignant & Sebaceous & 1 & $C$ & 70 \\
\hline $\mathrm{H} 6$ & 76 & $\mathrm{~F}$ & 20 & Ovary & Immature teratoma & $\|$ & Malignant & Immature & 1.5 & $C$ & 70 \\
\hline $\mathrm{H} 7$ & 77 & $\mathrm{~F}$ & 18 & Ovary & Immature teratoma & III & Malignant & Cystic epithelium & 1.5 & $C$ & 90 \\
\hline $\mathrm{H} 8$ & 78 & M & 45 & Ovary & Mature teratoma & - & Benign & Sebaceous & 2.5 & $C$ & 100 \\
\hline $\mathrm{H} 8$ & 78 & M & 45 & Ovary & Mature teratoma & - & Benign & Lymphocytes & 3 & $\mathrm{~N}$ & 50 \\
\hline $\mathrm{H} 9$ & 79 & $\mathrm{~F}$ & 47 & Ovary & Malignant teratoma & - & Benign & Apocrine & 1.5 & $C$ & 50 \\
\hline $\mathrm{H} 10$ & 80 & $\mathrm{~F}$ & 69 & Ovary & $\begin{array}{l}\text { Monodermal } \\
\text { teratoma }\end{array}$ & - & Malignant & Thyroid & 2.5 & C & 40 \\
\hline $\mathrm{H} 10$ & 80 & $\mathrm{~F}$ & 69 & Ovary & $\begin{array}{c}\text { Monodermal } \\
\text { teratoma }\end{array}$ & - & Malignant & Lymphocytes & 3 & $\mathrm{~N}$ & 30 \\
\hline
\end{tabular}

* Intensity was graded in the scale from 0 to 3; 0 - no staining, 1 - weak staining, 2 - moderate staining, 3 - strong staining.

** Location of the $\mathrm{CX}_{3} \mathrm{CR} 1$ staining was cytoplasmic $(\mathrm{C})$, nuclear $(\mathrm{N})$, and membranous $(\mathrm{M})$, as indicated.

*** Percentage of $\mathrm{CX}_{3} \mathrm{CR} 1$-positive cells is indicated.

\section{Methods}

\section{Materials}

Tissue microarray (TMA) slides containing specimens of ovarian teratoma (Cat\# OV805) and specimens of human fetal tissues (Cat\# BE01014) were obtained from US Biomax (Rockville, MD). Because these specimens were commercially available and were deidentified, no approvals by the Institutional Review Board were required. GFPtagged $\mathrm{CX}_{3} \mathrm{CR} 1$ shRNA and scrambled shRNA constructs were obtained from Origene Technologies (Rockville, MD). DharmaFECT was obtained from Dharmacon (Lafayette, $\mathrm{CO}$ ). Matrigel was obtained from BD Biosciences (Bedford, MA). Mouse monoclonal CK14 and CK18 and rabbit polyclonal $\mathrm{CX}_{3} \mathrm{CR} 1$ (Cat\# ab49747, ab49824, ab8021 and ab8020, respectively) antibodies were obtained from Abcam (Cambridge, MA), and mouse monoclonal $\beta$ tubulin antibody was obtained from Developmental Studies Hybridoma Bank (Iowa City, IA), goat polyclonal $\mathrm{CX}_{3}$ CL1 (Cat\# AF365) was purchased from R\&D systems (Minneapolis, MN), and mouse monoclonal actin antibody was purchased from Santa Cruz Biotechnology (Dallas, TX). Vectastain ABC and DAB kits were obtained from Vector Laboratories (Burlingame, CA). BMP-4 was purchased from Invitrogen (Carlsbad, CA).

\section{Cell culture and directed differentiation}

Ovarian teratocarcinoma cell line PA-1 and ovarian carcinoma cell line SKOV-3 were obtained from American Tissue Culture Collection (Manassas, VA). PA-1 cells were plated on Matrigel-coated (at 1/100 dilution) tissue culture plates or glass cover slips at $5 \%$ confluence and cultured in minimal essential media supplemented with sodium pyruvate and non-essential amino acids for 2 weeks. Cells subjected to differentiation into skin lineage were cultured in the above described media containing $10 \mu \mathrm{g} / \mathrm{ml} \mathrm{BMP}-4$.

\section{Immunohistochemical staining}

TMA slides were deparaffinized by baking at $60^{\circ} \mathrm{C}$ for $2 \mathrm{~h}$ and rehydrated by incubation in xylene, $100 \%$ ethanol, 95\% ethanol, 70\% ethanol, and phosphate buffered saline, $\mathrm{pH}$ 7.4, for 5 min each. Peroxidase activity was inhibited with hydrogen peroxide. Antigen retrieval was performed by $15 \mathrm{~min}$ incubation in $1 \mathrm{mM}$ ethylene diamine tetraacetic acid (EDTA; pH 8.0) at $95^{\circ} \mathrm{C}$. Prior to primary antibody staining (1:50 dilution, rabbit antihuman $\mathrm{CX}_{3} \mathrm{CR} 1$, Abcam Cat\# ab8021, $1 \mathrm{~h}$ at room temperature (RT)) non-specific binding was blocked by incubation with $10 \%$ goat serum for $1 \mathrm{~h}$. The biotinconjugated goat anti-rabbit secondary antibody was used at a 1:200 dilution for $30 \mathrm{~min}$ at RT. The Vectashield ABC kit was used as directed by the manufacturer, and tissues were incubated for $45 \mathrm{~min}$ at RT. The DAB reagent was prepared as instructed by the manufacturer and applied to tissues on TMA slides for 2 - 10 min until brown color developed. TMAs were stained with hematoxylin, dehydrated in 50\%, 70\%, 95\%, and 100\% ethanol, and mounted with Permount. Pancreatic cancer tissue was used as a positive 
control. Staining was evaluated by A.K.-B. and L.R., who were both blinded to the experimental outcomes of the study. Staining was scored based on the intensity and percentage of positive cells. Intensity of staining was " 0 " for negative samples, "1" for weakly positive samples, "2" for moderately positive samples, and " 3 " for highly positive samples. Overall scores were derived as the intensity score multiplied by the percentage. Staining was assessed separately in the cytoplasm and the membrane.

\section{Cell transfection and generation of stable cell lines}

PA-1 cells were cultured to $80 \%$ confluence and transfected with either $\mathrm{CX}_{3} \mathrm{CR} 1$-specific GFP-tagged shRNA construct or scrambled GFP-tagged shRNA construct (Origene Technologies) using DharmaFECT according to the manufacturer's instructions. Selection of the clones was performed with puromycin and green fluorescence as suggested by the manufacturer.

\section{Immunofluorescence staining}

Cells were cultured on glass coverslips to $50-70 \%$ confluence, fixed, permeabilized with $0.01 \%$ Triton X-100, and blocked in goat serum. Mouse monoclonal CK-14 (Cat\# ab49747) antibody was used at a dilution of 1:50. Cells were incubated with the primary antibodies for $1 \mathrm{~h}$ at room temperature $\left(22^{\circ} \mathrm{C}\right)$. The cells were then incubated with secondary Alexa555-conjugated anti-mouse antibodies $(1: 500)$ for $1 \mathrm{~h}$ at RT in the dark. 4,6Diamidino-2-phenylindole (DAPI) was added to the secondary antibody solution to a final concentration of $10 \mu \mathrm{g} / \mathrm{ml} 10 \mathrm{~min}$ prior to the end of the incubation period. Cells were washed, air dried, and mounted on glass slides using ProlongGold (Invitrogen, Carlsbad, CA). Fluorescent imaging was performed using a Zeiss AxioObserverD.1 fluorescent microscope.

\section{Western blotting}

Western blotting analysis was used to detect the expression of CK14, CK18, CX ${ }_{3}$ CR1, CX ${ }_{3}$ CL1, actin, and $\beta$-tubulin in PA-1. This procedure was performed as previously described $[8,14,15]$ Antibodies were used at the following dilutions: 1:500 mouse anti-human-CK14 or CK18, or $\mathrm{CX}_{3} \mathrm{CL} 1$, rabbit anti-human $\mathrm{CX}_{3} \mathrm{CR} 1$ (Abcam ab8020) and 1:200 mouse anti-human- $\beta$-tubulin or goat antihuman actin in $3 \%$ BSA in a solution of $50 \mathrm{mM}$ tris-buffered saline, $\mathrm{pH} 7.4,150 \mathrm{mM} \mathrm{NaCl}$, and $0.05 \%$ Tween-20 (TBST). Immunoreactive bands were visualized with anti-(mouse-IgG)-peroxidase, anti-(goat-IgG)peroxidase, and anti-(rabbit-IgG)-peroxidase (Sigma, St. Louis, MO) (1:1000 in 3\% BSA in TBST), and enhanced chemiluminescence was read using Chemidoc (Bio-Rad) and Bio-Rad Chemidoc ImageReader software.

\section{Results}

\section{$\mathrm{CX}_{3} \mathrm{CR} 1$ is expressed in specimens of ovarian teratoma}

According to our previous observations $\mathrm{CX}_{3} \mathrm{CR} 1$ was expressed in cells comprising ovarian teratoma tissue specimens [8], although the number of specimens was very limited in order to be able to draw any conclusions. In the present study we aimed to expand this observation on a larger number of ovarian teratoma specimens and determine the predominant cell type(s) positive for $\mathrm{CX}_{3} \mathrm{CR} 1$. Thus, we tested expression of $\mathrm{CX}_{3} \mathrm{CR} 1$ using a tissue microarray containing 68 specimens of mature ovarian teratoma, 10 specimens of immature teratoma, and 2 cases of monodermal teratoma. Interestingly, we have found that skin keratinocytes represent the most predominant $\mathrm{CX}_{3} \mathrm{CR} 1$-positive cell type, which was present in $38 \%$ of tested cases of mature ovarian teratoma (Table 1, Figure 1). The next most common $\mathrm{CX}_{3} \mathrm{CR} 1$-positive types of cells in the tested cases of dermoid cyst were those comprising the pilosebaceous unit of the skin assembled of sebaceous gland and hair follicle (Table 1, Figure 1).

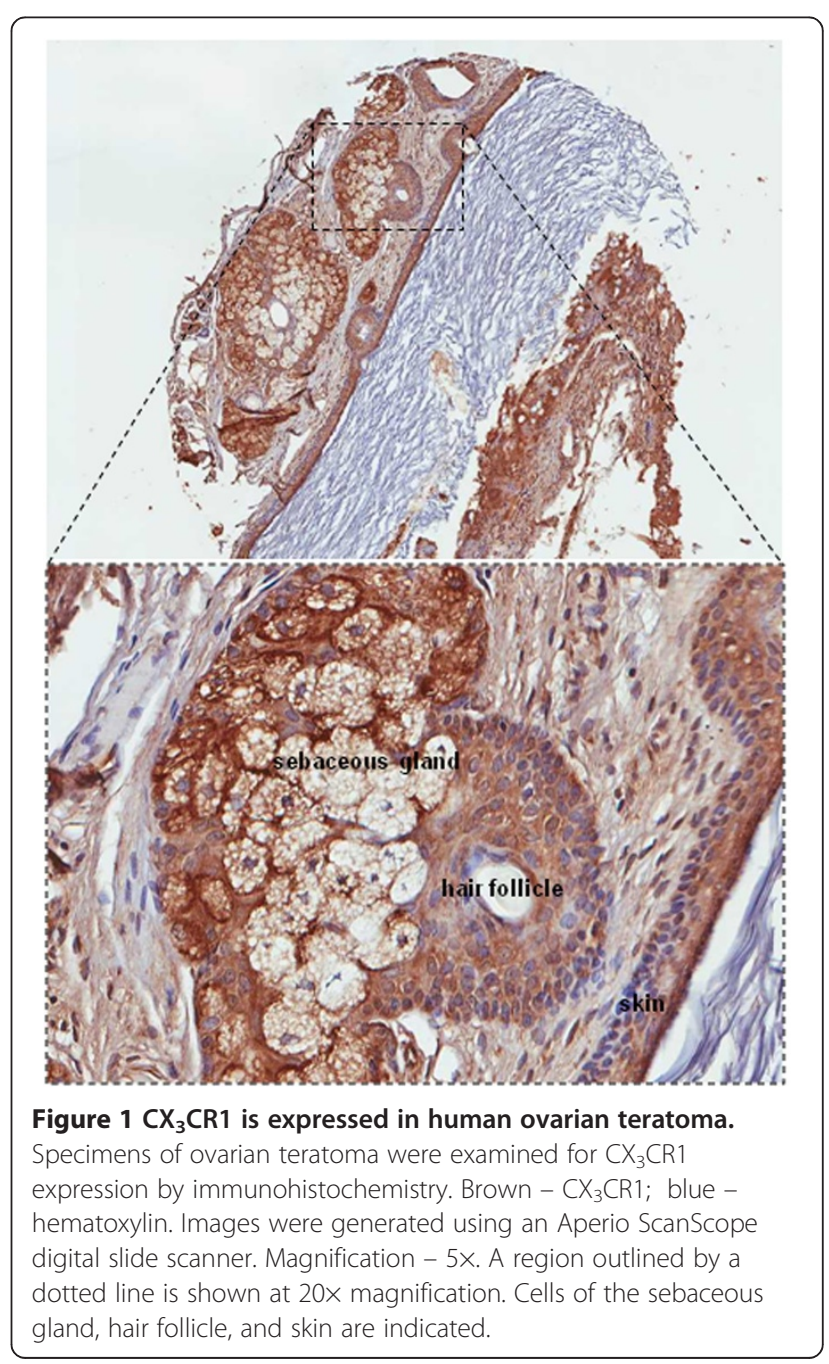


Table 2 Expression of CX3CR1 in human fetal tissues

\begin{tabular}{|c|c|c|c|c|c|c|c|c|c|c|}
\hline Position & Number & Sex* & Age $^{*}$ & Organ* & Type* & Intensity** & Location & Percentage $^{* * *}$ & Score ${ }^{* * * *}$ & Notes \\
\hline $\mathrm{A} 1$ & 1 & $M$ & 5 mon. & Heart & Normal & 2.5 & & 100 & 250 & \\
\hline A2 & 2 & M & 5 mon. & Heart & Normal & 2.5 & & 100 & 250 & \\
\hline A3 & 3 & M & 5 mon. & Heart & Normal & 2.5 & & 100 & 250 & \\
\hline A4 & 4 & M & 5 mon. & Gallbladder & Normal & 2 & & 100 & 200 & \\
\hline A5 & 5 & M & 5 mon. & Gallbladder & Normal & 2 & & 60 & 120 & \\
\hline A6 & 6 & M & 5 mon. & Gallbladder & Normal & 2 & & 90 & 180 & \\
\hline A7 & 7 & M & 5 mon. & Colon & Normal & 3 & & 100 & 300 & \\
\hline A8 & 8 & M & 5 mon. & Colon & Normal & 3 & & 100 & 300 & \\
\hline A9 & 9 & M & 5 mon. & Colon & Normal & 3 & & 100 & 300 & \\
\hline B1 & 10 & M & 5 mon. & Small intestine & Normal & 2.5 & & 100 & 250 & \\
\hline B2 & 11 & M & 5 mon. & Small intestine & Normal & 2.5 & & 100 & 250 & \\
\hline B3 & 12 & M & 5 mon. & Small intestine & Normal & 2.5 & & 100 & 250 & \\
\hline B4 & 13 & M & 5 mon. & Liver & Normal & 2.5 & & 100 & 250 & \\
\hline B5 & 14 & M & 5 mon. & Liver & Normal & 3 & & 100 & 300 & \\
\hline B6 & 15 & M & 5 mon. & Liver & Normal & 3 & & 100 & 300 & \\
\hline B7 & 16 & M & 5 mon. & Rectum & Normal & 2.5 & & 95 & 237.5 & \\
\hline B8 & 17 & M & 5 mon. & Rectum & Normal & 2 & & 95 & 190 & \\
\hline B9 & 18 & M & 5 mon. & Rectum & Normal & 2.5 & & 100 & 250 & \\
\hline C1 & 19 & M & 5 mon. & Stomach & Normal & 2 & & 70 & 140 & \\
\hline $\mathrm{C} 2$ & 20 & M & 5 mon. & Stomach & Normal & 1.5 & & 60 & 90 & \\
\hline C3 & 21 & M & 5 mon. & Stomach & Normal & 1.5 & & 80 & 120 & \\
\hline C4 & 22 & M & 5 mon. & Adrenal gland & Normal & 2.5 & & 80 & 200 & \\
\hline C5 & 23 & M & 5 mon. & Adrenal gland & Normal & 2 & & 90 & 180 & \\
\hline $\mathrm{C} 6$ & 24 & M & 5 mon. & Adrenal gland & Normal & 2 & & 60 & 120 & \\
\hline C7 & 25 & M & 5 mon. & Thyroid gland & Normal & 0.5 & & 30 & 15 & \\
\hline $\mathrm{C} 8$ & 26 & M & 5 mon. & Thyroid gland & Normal & 0.5 & & 10 & 5 & \\
\hline C9 & 27 & M & 5 mon. & Thyroid gland & Normal & 0.5 & & 10 & 5 & \\
\hline D1 & 28 & M & 5 mon. & Spleen & Normal & 1 & & 80 & 80 & \\
\hline D2 & 29 & M & 5 mon. & Spleen & Normal & 1 & & 80 & 80 & \\
\hline D3 & 30 & M & 5 mon. & Spleen & Normal & 1 & & 80 & 80 & \\
\hline D4 & 31 & M & 5 mon. & Thymus gland & Normal & 2.5 & $\begin{array}{l}\text { Epithelioid } \\
\text { cells }\end{array}$ & 20 & 50 & \\
\hline D4 & & & & & & 1 & Lymphocytes & 100 & 100 & \\
\hline D5 & 32 & M & 5 mon. & Thymus gland & Normal & 2.5 & $\begin{array}{l}\text { Epithelioid } \\
\text { cells }\end{array}$ & 20 & 50 & \\
\hline D5 & & & & & & 1 & Lymphocytes & 100 & 100 & \\
\hline D6 & 33 & M & 5 mon. & Thymus gland & Normal & 2.5 & $\begin{array}{l}\text { Epithelioid } \\
\text { cells }\end{array}$ & 20 & 50 & \\
\hline D6 & & & & & & 1 & Lymphocytes & 100 & 100 & \\
\hline D7 & 34 & M & 5 mon. & Skin & Normal & 2 & Epithelium & 80 & 160 & \\
\hline D7 & & & & & & 3 & Adipose & 70 & 210 & \\
\hline D8 & 35 & M & 5 mon. & Skin & Normal & 1.5 & Epithelium & 80 & 120 & \\
\hline D8 & & & & & & 3 & Adipose & 70 & 210 & \\
\hline D9 & 36 & M & 5 mon. & Skin & Normal & 2 & Epithelium & 90 & 180 & \\
\hline
\end{tabular}


Table 2 Expression of CX3CR1 in human fetal tissues (Continued)

\begin{tabular}{|c|c|c|c|c|c|c|c|c|c|c|}
\hline D9 & & & & & & 3 & Adipose & 80 & 240 & \\
\hline E1 & 37 & M & 5 mon. & Bone & Normal & 2.5 & & 30 & 75 & \\
\hline E2 & 38 & M & 5 mon. & Bone & Normal & 2.5 & & 30 & 75 & \\
\hline E3 & 39 & M & 5 mon. & Bone & Normal & 2.5 & & 30 & 75 & \\
\hline E4 & 40 & M & 5 mon. & Epididymis & Normal & 1 & & 90 & 90 & \\
\hline E5 & 41 & M & 5 mon. & Epididymis & Normal & 0.5 & & 60 & 30 & \\
\hline E6 & 42 & M & 5 mon. & Epididymis & Normal & 0.5 & & 70 & 35 & \\
\hline E7 & 43 & M & 5 mon. & Brain & Normal & 2 & & 20 & 40 & \\
\hline E8 & 44 & M & 5 mon. & Brain & Normal & 1 & & 5 & 5 & \\
\hline E9 & 45 & M & 5 mon. & Brain & Normal & 1 & & 5 & 5 & \\
\hline $\mathrm{F} 1$ & 46 & M & 5 mon. & Lung and trachea & Normal & 3 & Trachea & 90 & 270 & \\
\hline $\mathrm{F} 1$ & 46 & M & 5 mon. & Lung and trachea & Normal & 3 & Alveoli & 25 & 75 & \\
\hline $\mathrm{F} 2$ & 47 & M & 5 mon. & Lung and trachea & Normal & 3 & Trachea & 90 & 270 & \\
\hline $\mathrm{F} 2$ & 47 & M & 5 mon. & Lung and trachea & Normal & 1 & Alveoli & 25 & 25 & \\
\hline F3 & 48 & M & 5 mon. & Lung and trachea & Normal & 3 & Trachea & 80 & 240 & \\
\hline F3 & 48 & M & 5 mon. & Lung and trachea & Normal & 1 & Alveoli & 25 & 25 & \\
\hline $\mathrm{F} 4$ & 49 & M & 5 mon. & Muscle & Normal & 0.5 & & 100 & 50 & \\
\hline F5 & 50 & M & 5 mon. & Muscle & Normal & 0.5 & & 100 & 50 & \\
\hline F6 & 51 & M & 5 mon. & Muscle & Normal & 0.5 & & 100 & 50 & \\
\hline F7 & 52 & M & 5 mon. & Smooth muscle & Normal & 2 & & 100 & 200 & \\
\hline F8 & 53 & M & 5 mon. & Smooth muscle & Normal & 2 & & 100 & 200 & \\
\hline F9 & 54 & M & 5 mon. & Smooth muscle & Normal & 2 & & 100 & 200 & \\
\hline G1 & 55 & M & 5 mon. & Kidney & Normal & 2 & Tubules & 80 & 160 & \\
\hline G1 & 55 & M & 5 mon. & Kidney & Normal & 1 & Glomeruli & 50 & 50 & \\
\hline G2 & 56 & M & 5 mon. & Kidney & Normal & 2.5 & Tubules & 70 & 175 & \\
\hline G2 & 56 & M & 5 mon. & Kidney & Normal & 0.5 & Glomeruli & 30 & 15 & \\
\hline G3 & 57 & M & 5 mon. & Kidney & Normal & 1.5 & Tubules & 90 & 135 & \\
\hline G3 & 57 & M & 5 mon. & Kidney & Normal & 0.5 & Glomeruli & 70 & 35 & \\
\hline G4 & 58 & M & 5 mon. & Eye & Normal & 3 & & 100 & 300 & $\begin{array}{l}\text { Retinal pigment } \\
\text { epithelium }\end{array}$ \\
\hline G5 & 59 & M & 5 mon. & Eye & Normal & 3 & & 100 & 300 & $\begin{array}{l}\text { Retinal pigment } \\
\text { epithelium }\end{array}$ \\
\hline G6 & 60 & M & 5 mon. & Eye & Normal & 3 & & 100 & 300 & $\begin{array}{l}\text { Retinal pigment } \\
\text { epithelium }\end{array}$ \\
\hline G7 & 61 & $F$ & 5 mon. & Umbilical cord & Normal & 2 & & 100 & 200 & \\
\hline G8 & 62 & $\mathrm{~F}$ & 5 mon. & Umbilical cord & Normal & 1.5 & & 100 & 150 & \\
\hline G9 & 63 & $\mathrm{~F}$ & 5 mon. & Umbilical cord & Normal & NA & & NA & NA & \\
\hline $\mathrm{H} 1$ & 64 & $F$ & 5 mon. & Placenta & Normal & 0.5 & & 20 & 10 & \\
\hline $\mathrm{H} 2$ & 65 & $F$ & 5 mon. & Placenta & Normal & 0.5 & & 10 & 5 & \\
\hline $\mathrm{H} 3$ & 66 & $F$ & 5 mon. & Placenta & Normal & 0.5 & & 40 & 20 & \\
\hline $\mathrm{H} 4$ & 67 & $F$ & 5 mon. & Uterus & Normal & 0.5 & & 20 & 10 & \\
\hline $\mathrm{H} 5$ & 68 & $F$ & 5 mon. & Uterus & Normal & 0.5 & & 5 & 2.5 & \\
\hline $\mathrm{H} 6$ & 69 & $F$ & 5 mon. & Uterus & Normal & 0.5 & & 20 & 10 & \\
\hline $\mathrm{H} 7$ & 70 & $F$ & 5 mon. & Pancreas & Normal & 2 & $\begin{array}{l}\text { Acini and } \\
\text { ducts }\end{array}$ & 80 & 160 & \\
\hline
\end{tabular}


Table 2 Expression of CX3CR1 in human fetal tissues (Continued)

\begin{tabular}{|c|c|c|c|c|c|c|c|c|c|}
\hline $\mathrm{H} 8$ & 71 & $F$ & 5 mon. & Pancreas & Normal & 2.5 & $\begin{array}{c}\text { Acini and } \\
\text { ducts }\end{array}$ & 80 & 200 \\
\hline H9 & 72 & $\mathrm{~F}$ & 5 mon. & Pancreas & Normal & 2.5 & $\begin{array}{c}\text { Acini and } \\
\text { ducts }\end{array}$ & 70 & 175 \\
\hline 11 & 73 & $\mathrm{~F}$ & 5 mon. & Ovary & Normal & 3 & & 70 & 210 \\
\hline 12 & 74 & $\mathrm{~F}$ & 5 mon. & Ovary & Normal & 3 & & 80 & 240 \\
\hline 13 & 75 & $F$ & 5 mon. & Ovary & Normal & 3 & & 90 & 270 \\
\hline 14 & 76 & $\mathrm{~F}$ & 5 mon. & Fallopian tube & Normal & 0.5 & & 5 & 2.5 \\
\hline 15 & 77 & $\mathrm{~F}$ & 5 mon. & Fallopian tube & Normal & 1 & & 10 & 10 \\
\hline 16 & 78 & $\mathrm{~F}$ & 5 mon. & Fallopian tube & Normal & 1.5 & & 5 & 7.5 \\
\hline
\end{tabular}

* Information obtained from the manufacturer (US Biomax; Rockville, MD) for the multiple organ fetal tissue array BE01014; TMA contained triplicate cores (26cases/78cores).

** Intensity of staining was scored as negative (0), weak positive (1), moderate positive (2), and strong positive (3).

*** Denotes percentage of positive cells in the core.

**** Score has been calculated as intensity multiplied by percentage of positive cells.

Other $\mathrm{CX}_{3}$ CR1-positive cell types in the order of diminution included lymphatic, apocrine, adipose, thyroid, respiratory epithelial, peripheral nervous, endothelial, and eccrine (Table 1). Cases of tested immature ovarian teratoma were characterized by the presence of $\mathrm{CX}_{3} \mathrm{CR} 1$-positive brain, lymphatic, respiratory epithelial, adipose, and sebaceous cells (Table 1). Two tested monodermal teratomas consisted of $\mathrm{CX}_{3} \mathrm{CR} 1$-positive thyroid and lymphatic tissues, respectively (Table 1 ). Thus, our data suggest that skin keratinocytes are the predominant type of $\mathrm{CX}_{3} \mathrm{CR} 1$-positive cells in the tested specimens of mature ovarian teratomas.

\section{$\mathrm{CX}_{3} \mathrm{CR} 1$ is expressed in fetal tissues}

Further we wished to determine $\mathrm{CX}_{3} \mathrm{CR} 1$-positive cells undergoing the normal development. We tested both male and female fetal tissues aged 5 months in utero. We found that the majority of epithelial cells, including skin keratinocytes, were $\mathrm{CX}_{3} \mathrm{CR} 1$-positive (Table 2). Hence, $\mathrm{CX}_{3} \mathrm{CR} 1$-positive skin cells are present not only within abnormally differentiated tissues, such as mature ovarian teratomas, but also in the normally developing skin.

\section{$\mathrm{CX}_{3} \mathrm{CR} 1$ is required for keratinocyte differentiation}

Based on our observations regarding $\mathrm{CX}_{3} \mathrm{CR} 1$ expression in both normally and abnormally developing keratinocytes, we hypothesized that $\mathrm{CX}_{3} \mathrm{CR} 1$ may be important for cell differentiation into the epidermal lineage. Thus, we tested the requirement for $\mathrm{CX}_{3} \mathrm{CR} 1$ in keratinocyte differentiation using a cell culture model. Ovarian teratocarcinoma cell line PA-1 has been established from the ascites of a patient with malignant ovarian teratoma [16]. Chromosomal analysis had suggested that these cells have a heterozygous origin and derived at a stage prior to the first meiotic division [17]. Several studies have shown that PA-1 is capable of differentiation into diverse cell lineages at the appropriate culture conditions $[16,18,19]$. Based on many similarities between the teratocarcinoma cells and early embryonic cells, the former has been used to study processes of differentiation $[19,20]$. Using gene specific shRNA constructs, we downregulated $\mathrm{CX}_{3} \mathrm{CR} 1$ in PA-1 cell line (Figure 2A). Further we cultured both $\mathrm{CX}_{3} \mathrm{CR} 1$ sh-PA-1 and the control cells transfected with a scrambled shRNA construct on Matrigel in the presence and absence of $0.5 \mathrm{nM}$ BMP-4 for 2 weeks. Expression of cytokeratin 14 (CK14) and cytokeratin 18 (CK18) was analysed with immunofluorescence microscopy and Western blotting (Figure 2B,C). We observed that only PA-1 transfected with scrambled shRNA and cultured in BMP-4-supplemented media expressed significantly higher levels of CK14 compared to scrambled shRNA PA- 1 or $\mathrm{CX}_{3} \mathrm{CR} 1$ shRNA PA1cultured in the absence of BMP-4, as well as $\mathrm{CX}_{3} \mathrm{CR} 1$ shRNA PA-1 cultured in the presence of BMP-4 (Figure 2B,C). In opposite, scrambled shRNA PA-1 or $\mathrm{CX}_{3} \mathrm{CR} 1$ shRNA PA-1cultured in the absence of BMP-4, as well as $\mathrm{CX}_{3} \mathrm{CR} 1$ shRNA PA-1 cultured in the presence of BMP-4 expressed higher levels of CK18 compared to scrambled shRNA PA-1 cultured in the presence of BMP-4 (Figure 2C). PA-1 expressed fractalkine ligand $\left(\mathrm{CX}_{3} \mathrm{CL} 1\right)$ necessary for the receptor $\left(\mathrm{CX}_{3} \mathrm{CR} 1\right)$ activation (Figure $\left.2 \mathrm{D}\right)$. These data suggest that $\mathrm{CX}_{3} \mathrm{CR} 1$-positive PA-1 cells are able to proceed toward differentiation into skin keratinocytes in the presence of BMP-4 and begin expressing a marker of stratified epithelium CK14, while the loss of $\mathrm{CX}_{3} \mathrm{CR} 1$ restricts differentiation and the cells remain CK18-positive simple-epithelial cells.

\section{Discussion}

Our findings demonstrate that cells of the skin lineage comprise the most predominant $\mathrm{CX}_{3} \mathrm{CR} 1$-positive cell type within the specimens of ovarian teratoma. Mature skin is a predominant tissue found in mature ovarian teratomas [21]. Our findings also show that normally developed keratinocytes are $\mathrm{CX}_{3} \mathrm{CR} 1$-positive. Importantly, 


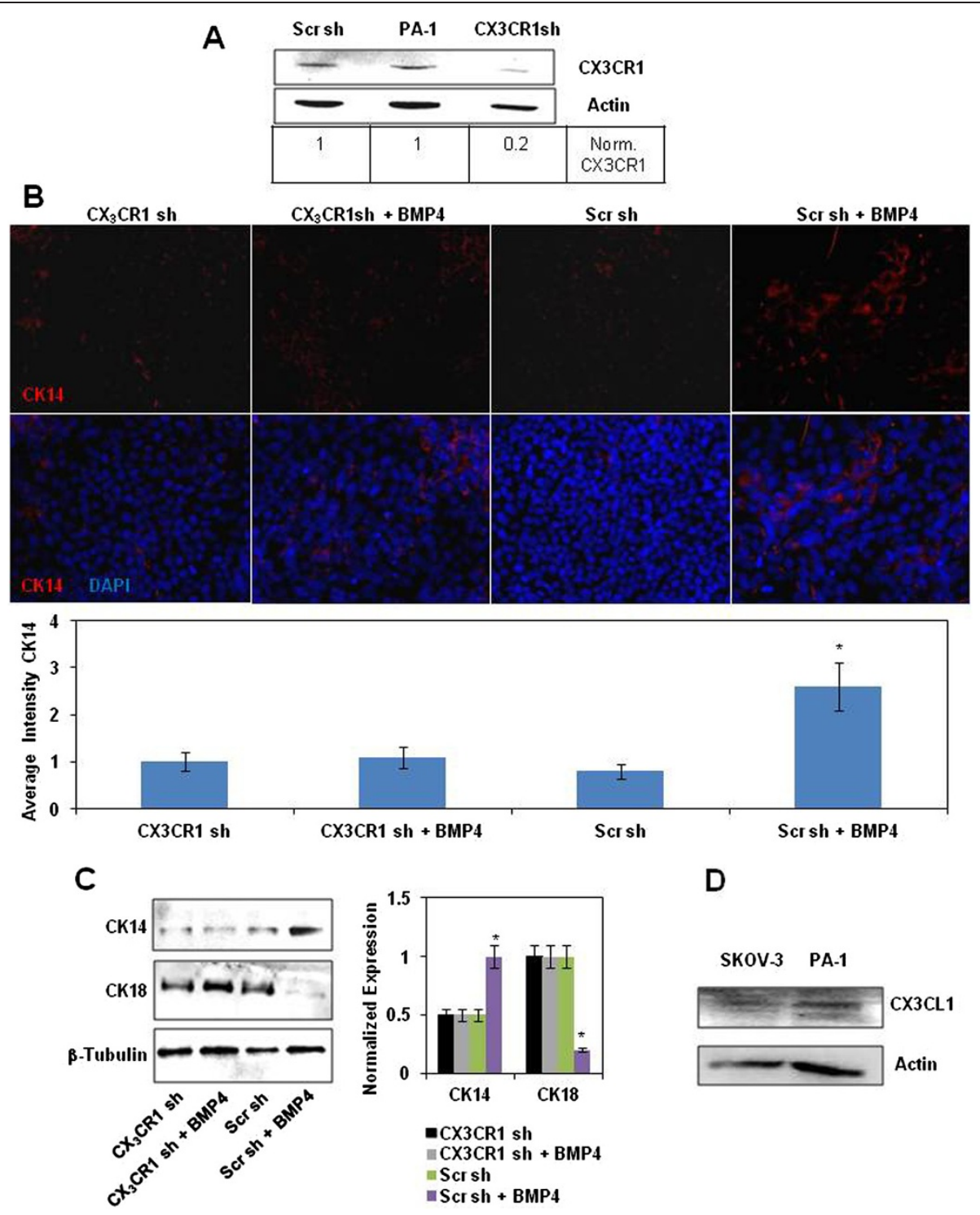

Figure $2 \mathrm{CX}_{3} \mathrm{CR} 1$ is required for keratinocytes differentiation. (A) Expression of $C X_{3} C R 1$ in parental PA-1 cell line and that stably transfected with either $C X_{3} C R 1$ or scrambled shRNA constructs was examined with Western blot. Actin served as a loading control. $C X_{3} C R 1$ expression levels were quantified using digital densitometry and normalized to the levels of actin expression. (B) Expression of cytokeratin 14 in PA-1 cells stably transfected with either $\mathrm{CX}_{3} \mathrm{CR} 1$ or scrambled shRNA constructs treated with recombinant BMP-4 or vehicle, as indicated, was examined with immunocytochemistry. CK14 in PA-1 was probed with anti-CK14 and Alexa555-conjugated anti-rabbit antibodies, and DNA was stained with 4',6Diamidino-2-Phenylindole, Dihydrochloride (DAPI); CK14 - red, DNA - blue. Images were taken using $5 \times$ magnification on the objective. The histogram demonstrates the average intensity of CK14 signal across the field as determined by the Zeiss AxioVision software. Data is an average of three independent experiments. ${ }^{*} p<0.05$. (C) Expression of cytokeratins 14 and 18 in PA-1 cells stably transfected with either CX $C_{3}$ CR1 or scrambled shRNA constructs treated with recombinant BMP-4 or vehicle, as indicated, was examined with Western blot. $\beta$-Tubulin served as a loading control. The histogram shows CK14 and CK18 expression levels. Expression of CK18 in CX ${ }_{3}$ CR1shRNA-transfected PA-1 cells was arbitrarily set as 1 and expression of both CK14 and CK18 in other conditions was calculated accordingly. The data represent a typical Western blot image, and quantitative analysis was performed using three independently performed experiments. ${ }^{*} p<0.05$. (D) Expression of $C X_{3} C L 1$ in $S K O V-3$ and PA-1 cell lines was examined with Western blot. Actin served as a loading control. SKOV-3 cell line was used as a positive control.

our findings suggest that $\mathrm{CX}_{3} \mathrm{CR} 1$ could be an important player in keratinocyte differentiation. Our data indicate that teratocarcinoma cell line PA-1 expressed a basal keratinocyte marker CK14 when cultured on Matrigel in the presence of BMP-4. In opposite, cells with silenced $\mathrm{CX}_{3} \mathrm{CR} 1$ and those cultured in the absence of BMP-4 expressed CK18 specific for simple nonstratified epithelium.

The role for $\mathrm{CX}_{3} \mathrm{CR} 1$ in skin differentiation has not been described before, although, the role of this receptor 
in skin wound healing has been reported [22]. Our findings could be potentially important, as skin is the largest organ of the human body. Many pathological conditions and injuries require regeneration of the skin, and affecting the $\mathrm{CX}_{3} \mathrm{CR} 1$-dependent pathway could be one way to achieving faster skin formation from cells capable of differentiation. Thus, the studies of the role of $\mathrm{CX}_{3} \mathrm{CR} 1$ in keratinocyte differentiation need to be expanded further to include more extensive studies on other abnormally differentiating teratoma cell lines, as well as normally differentiating embryonic stem cells. Furthermore, we observed that CX3CR1 is expressed in other developing tissues, as indicated in Table 2, thus, it is possible that this receptor could be important for the development of those tissues as well. In fact, a role for the fractalkine axis in osteoclasts differentiation has been reported, whereby $\mathrm{CX}_{3} \mathrm{CL} 1$-expressing osteoblasts regulate differentiation of $\mathrm{CX}_{3} \mathrm{CR} 1$ positive osteoclast precursors [12]. Hence, more rigorous future studies of the role of $\mathrm{CX}_{3} \mathrm{CR} 1$ in development and differentiation are required.

\section{Conclusions}

Here we present that fractalkine receptor is expressed by skin cells in specimens of human ovarian teratoma and fetus and is required for epidermal lineage differentiation. This information sheds light on the ovarian pathology, dermoid cyst, and outlines possible mechanisms of cell differentiation within this benign formation. As the fractalkine receptor has recently emerged as a novel potential therapeutic target against many debilitating diseases, it is important to remember its role in cell differentiation when applying future antifractalkine therapies.

\section{Abbreviations}

BMP-4: Bone morphogenic protein 4; BSA: Bovine serum albumin; CK14: Cytokeratin 14; CK18: Cytokeratin 18; CX ${ }_{3}$ CL1: Fractalkine (chemokine ligand); $\mathrm{CX}_{3} \mathrm{CR}$ : Fractalkine receptor (chemokine receptor); DAPI: 4',6diamidino-2-phenylindole; EDTA: Ethylenediaminetetraacetic acid; GFP: Green fluorescent protein; PA-1: Ovarian teratocarcinoma cell line; TMA: Tissue microarray.

\section{Competing interests}

All authors declare neither financial nor non-financial competing interests.

\section{Authors' contributions}

$L R$ analysed $C X_{3} C R 1$ expression in tissue microarrays and interpreted the data. $H G$ analysed expression of $C X_{3} C R 1$ and $C X_{3} C L 1$. AAKB analysed $C X_{3} C R 1$ expression in tissue microarrays, interpreted the data, and contributed to study design. MVB conceived of the study, performed immunohistochemical staining, created stable clones with shRNA, performed immunofluorescence and Western blot experiments, and drafted the manuscript. All authors read and approved the final manuscript.

\section{Authors' information}

AAKB is a pathologist with specialization in gynecologic oncology. MVB is a basic cancer researcher whose main research interests are on metastatic ovarian carcinoma. Both AAKB and MVB share research interests on the role of $C X_{3} C L 1 / C X_{3} C R 1$ axis in prostate and ovarian cancers, respectively. $L R$, $A A K B$, and MVB have recently co-authored an article describing a role of $\mathrm{CX}_{3} \mathrm{CR} 1$ in progression of metastatic ovarian carcinoma. This current study seeks to expand the understanding of the biological impact of the fractalkine axis on cell differentiation.

Study design: fractalkine receptor is expressed by skin cells in specimens of human ovarian teratoma and fetus and is required for epidermal lineage differentiation.

\section{Acknowledgements}

Sources of financial support: American Cancer Society, Illinois Division, grant \#198484 (to MVB), NIH National Cancer Institute, grant \#1R21CA160917 (to MVB), and Liz Tilberis Award from the Ovarian Cancer Research Fund (to MVB).

\section{Author details}

${ }^{1}$ Departments of Pathology, University of Illinois at Chicago, $833 \mathrm{~S}$ Wood Str Chicago, IL 60612, USA. ${ }^{2}$ Biopharmaceutical Sciences, University of Illinois at Chicago, 833 S Wood Str, Chicago, IL 60612, USA.

Received: 11 June 2013 Accepted: 14 August 2013

Published: 17 August 2013

\section{References}

1. Prat J: Female reproductive system. In Anderson's pathology. Edited by Damjanov ILJ. St. Louis: Mosby; 1996:2231-2309.

2. Gasim T, Al Dakhiel SA, Al Ghamdi AA, Al Ali M, Al Jama F, Rahman J, Al Suleiman SA, Rahman MS: Ovarian tumors associated with pregnancy: a 20-year experience in a teaching hospital. Arch Gynecol Obstet 2010, 282(5):529-533.

3. Fishman A, Edelstein E, Altaras M, Beyth Y, Bernheim J: Adenocarcinoma arising from the gastrointestinal epithelium in benign cystic teratoma of the ovary. Gynecol Oncol 1998, 70(3):418-420.

4. Kido A, Togashi K, Konishi I, Kataoka ML, Koyama T, Ueda H, Fujii S, Konishi J: Dermoid cysts of the ovary with malignant transformation: MR appearance. AJR Am J Roentgenol 1999, 172(2):445-449.

5. Parrington JM, West LF, Povey S: The origin of ovarian teratomas. J Med Genet 1984, 21(1):4-12.

6. Outwater EK, Siegelman ES, Hunt JL: Ovarian teratomas: tumor types and imaging characteristics. Radiographics 2001, 21(2):475-490.

7. Rha SE, Byun JY, Jung SE, Kim HL, Oh SN, Kim H, Lee H, Kim BK, Lee JM: Atypical CT and MRI manifestations of mature ovarian cystic teratomas. AJR Am J Roentgenol 2004, 183(3):743-750.

8. Kim M, Rooper L, Xie J, Kajdacsy-Balla AA, Barbolina MV: Fractalkine receptor CX(3)CR1 is expressed in epithelial ovarian carcinoma cells and required for motility and adhesion to peritoneal mesothelial cells. Mol Cancer Res 2012, 10(1):11-24.

9. D'Haese JG, Demir IE, Friess H, Ceyhan GO: Fractalkine/CX3CR1: why a single chemokine-receptor duo bears a major and unique therapeutic potential. Expert Opin Ther Targets 2010, 14(2):207-219.

10. D'Haese JG, Friess H, Ceyhan GO: Therapeutic potential of the chemokinereceptor duo fractalkine/CX3CR1: an update. Expert Opin Ther Targets 2012, 16(6):613-618.

11. Imai T, Hieshima K, Haskell C, Baba M, Nagira M, Nishimura M, Kakizaki M, Takagi S, Nomiyama H, Schall TJ, Yoshie O: Identification and molecular characterization of fractalkine receptor CX3CR1, which mediates both leukocyte migration and adhesion. Cell 1997, 91(4):521-530.

12. Koizumi K, Saitoh Y, Minami T, Takeno N, Tsuneyama K, Miyahara T, Nakayama T, Sakurai H, Takano Y, Nishimura M, Imai T, Yoshie O, Saiki I: Role of $\mathrm{CX} 3 \mathrm{CL} 1 /$ fractalkine in osteoclast differentiation and bone resorption. J Immunol 2009, 183(12):7825-7831.

13. Łyszkiewicz M, Witzlau K, Pommerencke J, Krueger A: Chemokine receptor CX3CR1 promotes dendritic cell development under steady-state conditions. Eur J Immunol 2012, 41(5):1256-1265.

14. Barbolina MV, Kim M, Liu Y, Shepard J, Belmadani A, Miller RJ, Shea LD, Stack MS: Microenvironmental regulation of chemokine (C-X-C-motif) receptor 4 in ovarian carcinoma. Mol Cancer Res 2010, 8(5):653-664.

15. Kim M, Rooper L, Xie J, Rayahin J, Burdette JE, Kajdacsy-Balla AA, Barbolina MV: The lymphotactin receptor is expressed in epithelial ovarian carcinoma and contributes to cell migration and proliferation. Mol Cancer Res 2012, 10(11):1419-1429.

16. Giovanella BC, Stehlin JS, Williams LJ Jr: Heterotransplantation of human malignant tumors in "nude" thymusless mice. II. Malignant tumors 
induced by injection of cell cultures derived from human solid tumors. J Natl Cancer Inst 1974, 52(3):921-930.

17. Zeuthen J, Nørgaard JO, Avner P, Fellous M, Wartiovaara J, Vaheri A, Rosén A, Giovanella BC: Characterization of a human ovarian teratocarcinomaderived cell line. Int J Cancer 1980, 25(1):19-32.

18. Xia SL, Zhang $X$, Jing $\mathrm{NH}$ : The induction and differentiation of a human teratocarcinoma cell line (PA-1) in vitro]. Shi yan sheng wu xue bao 1995, 28(4):397-407.

19. Zeuthen J: Human teratocarcinoma cell lines. A review. Workshop on early detection of testicular cancer. Copenhagen, Denmark. Int J Androl 1981, Supplementum 4:61-77.

20. Illmensee K, Mintz B: Totipotency and normal differentiation of single teratocarcinoma cells cloned by injection into blastocysts. Proc Natl Acad Sci USA 1976, 73(2):549-553.

21. Saba L, Guerriero S, Sulcis R, Virgilio B, Melis G, Mallarini G: Mature and immature ovarian teratomas: $\mathrm{CT}$, US and MR imaging characteristics. Eur J Radiol 2009, 72(3):454-463.

22. Ishida Y, Gao JL, Murphy PM: Chemokine receptor CX3CR1 mediates skin wound healing by promoting macrophage and fibroblast accumulation and function. J Immunol 2008, 180(1):569-579.

doi:10.1186/1757-2215-6-57

Cite this article as: Rooper et al:: Fractalkine receptor is expressed in mature ovarian teratomas and required for epidermal lineage differentiation. Journal of Ovarian Research 2013 6:57.

\section{Submit your next manuscript to BioMed Central and take full advantage of:}

- Convenient online submission

- Thorough peer review

- No space constraints or color figure charges

- Immediate publication on acceptance

- Inclusion in PubMed, CAS, Scopus and Google Scholar

- Research which is freely available for redistribution 